\title{
A Reappraisal of the Development of the Eyelids
}

\author{
D. SEVEL \\ La Jolla, California
}

\begin{abstract}
Summary
The eyelids develop from ectoderm and from mesoderm. This evolution passes through distinct phases of maturation. Contemporaneously, the anterior segment of the eye takes form. The role of the amniotic fluid in the development of the eyelids is considered.

The purpose of this report is to describe the development of the eyelids. The evolution of the eyelids from early embryonic life through different stages of development to term is examined.
\end{abstract}

\section{Materials and Methods}

Sixty-four human eyes (Fig. 1) from embryos and fetuses ranging in size from $13.6 \mathrm{~mm}$ to term were examined. ${ }^{1,2}$ Between 60 and 110 microscopic sections were examined, respectively, from each embryo and fetus. The normal development of the embryo/fetus was an essential prerequisite, so the embryo/fetus from septic abortion was not accepted for investigation. If the mother had been exposed to teratogenic drugs during pregnancy, the embryo or fetus was excluded from the survey. Where salpingectomy was done (for ectopic pregnancy), the fallopian tubes were dissected with the aid of a dissecting microscope and the embryos retrieved. Usually these embryos were found within blood clots.

\section{Age of Embryo and Fetus}

The maternal history, with reference to duration of pregnancy, was found to be unreliable. A more satisfactory correlation was found between the clinical size of the uterus and the age of the embryo/fetus. Where possible, however, a correlation was attempted between the clinical age of the embryo/fetus and its crown-rump length. A micrometre gauge was used for measuring the length of the fetus up to $170 \mathrm{~mm}$; thereafter a centimeter metal rule was used. Development of the eye was also correlated with development of other body organs. It was then possible to assess this interrelationship, and therefore assign an age to the embryo-fetus. ${ }^{3,4}$ Specimens were examined microscopically with a dissecting microscope. Small specimens (less than $80 \mathrm{~mm}$ crown/rump length) were fixed for at least one week and larger specimens (larger than $80 \mathrm{~mm}$ crown/rump length) for at least two weeks.

All specimens were serially sectioned in conventional anatomic planes, either frontal (coronal) or transverse (horizontal). Stains used on the sections were heamatoxylineosin, Masson trichrome, Mallory's phosphogtungstic acid heamatoxylin, Verhoeff's van Gieson elastic, Bodian's silver nitrate and Luxol fast blue.

\section{Results}

It was noted that the eyelids pass through five stages of development:

(1) A stage of eyelid folds $(15 \mathrm{~mm}$ to $35 \mathrm{~mm}-6$ weeks to 8 weeks);

(2) a stage of eyelid fusion (35 $\mathrm{mm}$ to $175 \mathrm{~mm}-8$ weeks to 5 months); 
D. SEVEL

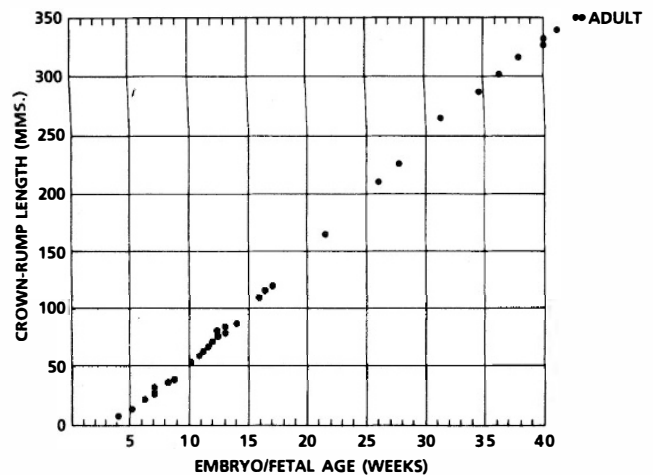

Fig. 1. Crown-rump length ( $\mathrm{mm}$ ) and age (weeks) of embryos and fetuses examined.

(3) a stage of development of specialised structures of the eyelids $(35 \mathrm{~mm}$ to $250 \mathrm{~mm}-8$ weeks to 7 months);

(4) a stage of eyelid separation ( $175 \mathrm{~mm}-$ $250 \mathrm{~mm}-5$ months to 7 months);

(5) a stage of eyelid maturation $(250 \mathrm{~mm}$ to term).

Up to the size of approximately $15 \mathrm{~mm}$, the optic vesicles are bathed in amniotic fluid. The cornea prior to the lid fusing is covered by three to four layers of epithelium, which are continuous with the surface ectoderm.

Stage of lid folds $(15 \mathrm{~mm}$ to $35 \mathrm{~mm}, 6$ weeks to 8 weeks).

At about $15 \mathrm{~mm}$ in size, the upper lid folds develop superiorally from a proliferation of mesodermal cells from the medial and lateral protuberances extending from the frontal nasal process (Fig. 2). The lower lid develops from a proliferation of mesodermal cells arising from the maxillary process (visceral

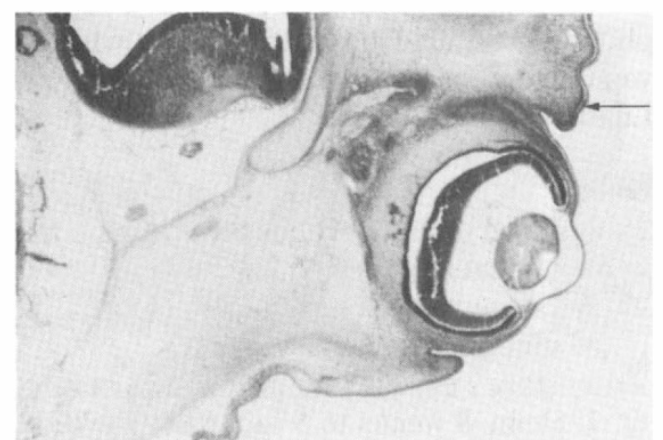

Fig. 2. Eyelid fold (arrow) developing from frontal nasal process $(30 \mathrm{~mm}$, Heamatoxylin-eosin $\times 3)$. mesoderm). These folds grow towards one another. They are covered by epithelium on their respective anterior and posterior surfaces. There are two layers of epithelium on the anterior surface and a single layer of epithelium on the posterior surface of the lid fold. The posterior single layer of epithelium becomes modified to form the tarsal conjunctiva and merges at the fornices with the bulbar conjunctiva.

Stage of eyelid fusion $35 \mathrm{~mm}$ to $175 \mathrm{~mm}, 8$ weeks to 5 months (Fig. 3).

The superior and inferior lid folds elongate and the epithelial margins fuse just anterior to the horizontal meridian of the eye (Fig. 4). A rather shallow conjunctival cavity results. At the site of fusion there are four opposing layers of epithlium; two layers of superficial epithelium from the upper lid and two from the lower lid. Contemporaneously with the fusion of the lids, an orchestrated development of morphological changes is triggered in the anterior segment of the eye.

Stage of development of specialised structures of the lids (35 mm to $250 \mathrm{~mm}-8$ weeks to 7 months).

It is during the stage of lid fusion that the specialised structures of the lid develop. These include:

(1) the orbicularis oculi muscle (Fig. 5);

(2) the tarsal plates and meibomian glands;

(3) the lacrimal puncta and canaliculi;

(4) the skin appendages;

(5) the conjunctiva.

The orbicularis oculi muscle develops from local mesenchymal tissue of the second vis-

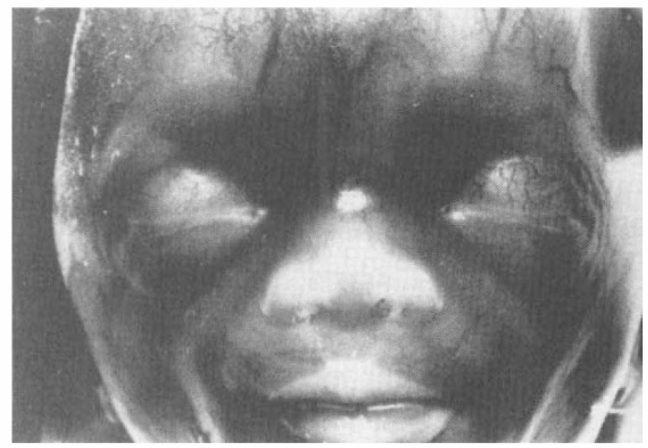

Fig. 3. Lids fused in 4 month fetus. 


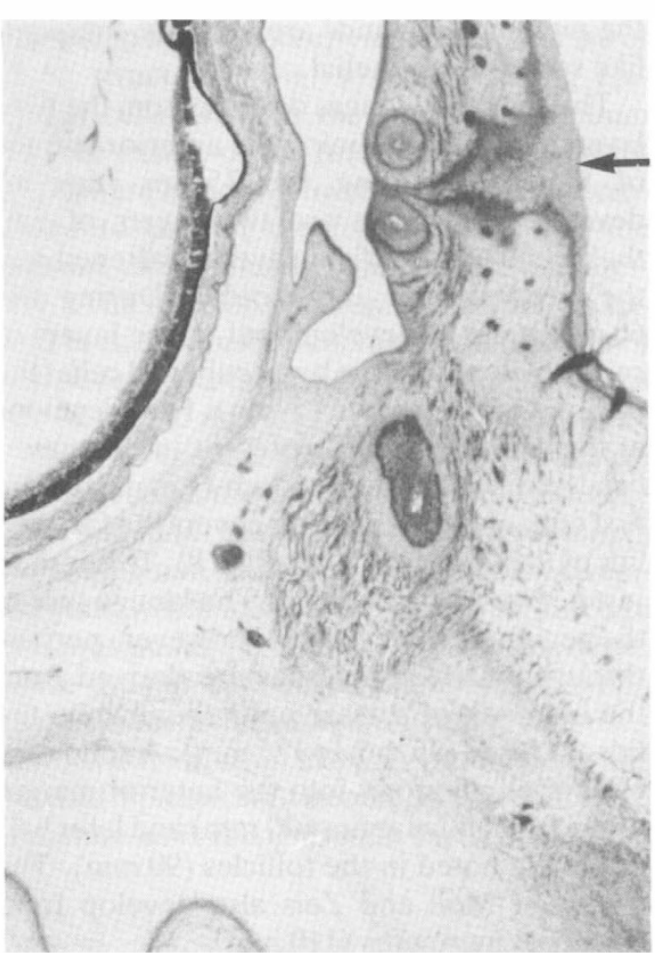

Fig. 4. Fusion (arrow) of upper and lower eyelid folds $(80 \mathrm{~mm}$, Heamatoxylin-eosin $\times 3)$.

ceral arch and develops in a similar manner to that of the extraocular muscles. The stages of muscle development include the stage of mesenchymal cell (up to $36 \mathrm{~mm}$ size embryo), stage of early myoblast formation $(13.6 \mathrm{~mm}$ size embryo), the myoblast stage (from $38.2 \mathrm{~mm}$ size embryo), the fusion of myoblast stage (from $54 \mathrm{~mm}$ size embryo), the myotube stage (from $54 \mathrm{~mm}$ size embryo), and mature

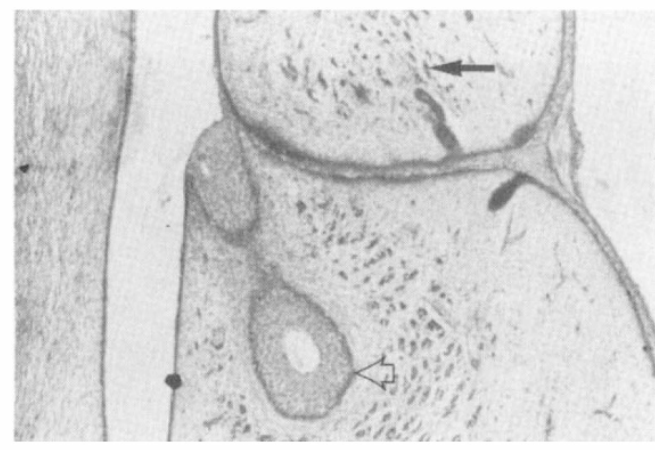

Fig. 5. Orbicularis oculi muscle (arrow) develops in lid and surrounds canaliculi (open arrow) $(180 \mathrm{~mm}$ Heamatoxylin-eosin $\times 3.5$ ). muscle stage (from $80.0 \mathrm{~mm}$ size fetus.) ${ }^{1}$ The sphincter-like orbicularis oculi muscle appears as a sheet covering the entire anterior surface of the lids during the fusional stage of development. The pretarsal, preseptal and orbital parts of the orbicularis oculi muscles are noted more distinctly at the $250 \mathrm{~mm}$ stage of development. With separation of the upper and the lower lids this sheet-like muscle is divided. The muscle extends medially to be attached to the medial palpebral ligament and also to the lateral palpebral ligament (vide infra).

The anlage of the tarsal plate is first noted at about the $40 \mathrm{~mm}$ stage of development. It develops from local mesenchymal tissue in the lid which becomes thickened and more compact. The tarsal plate lies posterior to the developing orbicularis oculi muscle. This compacted mesenchymal tissue extends from the eyelid margin to the site where the levator palpebrae superioris muscle develops at the superior margin of the lid $(40 \mathrm{~mm})$. The levator palpebrae superioris muscle passes through similar phases of development to those of the extra ocular muscles. ${ }^{1}$ The levator palpebrae superioris muscle, the superior rectus muscle, and the superior oblique muscles, develop from the same mesodermal complex, i.e., the superior mesodermal complex (Figs $6,7) \cdot{ }^{1,6,7}$ By a process of differential growth and degeneration, these muscles separate.

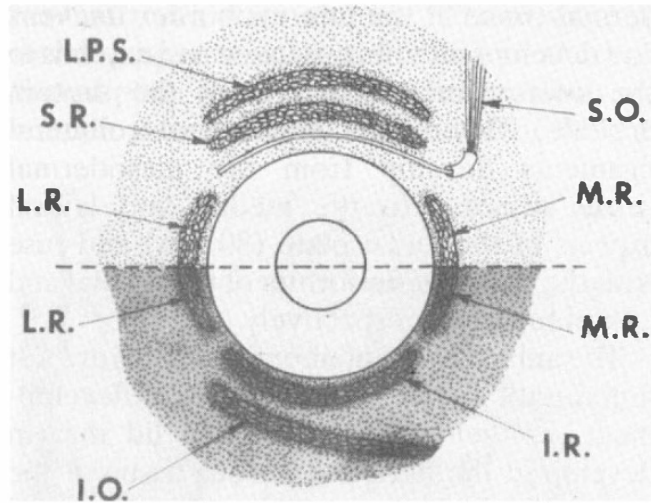

Fig. 6. Levator palpebrae superioris (LPS), superior rectus (SR), superior oblique (SO), part of laterarl rectus (LR) and part of medial rectus (MR) muscles arise from the superior mesodermal complex (light stipple); inferior rectus (IR), part of medial rectus (MR), and part of lateral rectus (LR) muscles arise from the inferior mesodermal complex (dark stipple). 


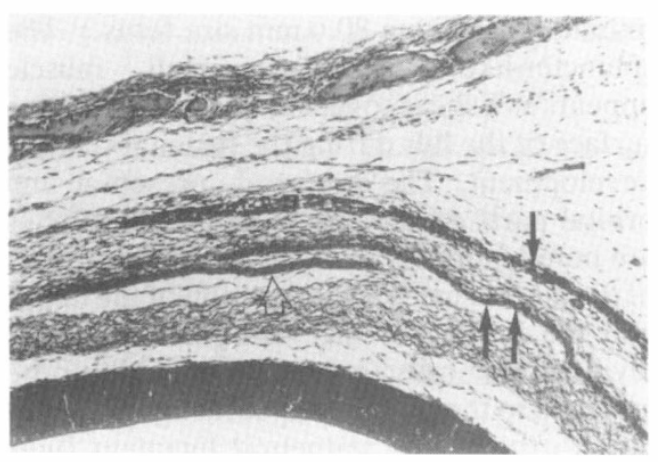

Fig. 7. The levator palpebrae superiortendon (arrow) differentiates from the superior rectus (double arrow) and the superior oblique (open arrow) tendons. All three tendons are derived from the superior mesodermal complex $(60 \mathrm{~mm}$, Heamatoxylin-eosin $\times 2.5)$.

With growth and maturation, the superior tarsal plate becomes D-shaped, the horizontal inferior border becoming the inferior lid margin. The levator palpebrae superioris muscle, merges with the convex superior border, with the anterior surface of the tarsal plate $(60 \mathrm{~mm})$ and with the skin anteriorly via septae which pass through the substance of the orbicularis oculi muscle and fuse with the superficial epidermal layer $(80 \mathrm{~mm})$ forming the superior tarsal sulcus.

The inferior tarsal plate develops pari passu with that of the upper lid $(40 \mathrm{~mm})$, however, with maturation it remains sausage-shaped. In man, there is no muscle attached to the inferior border of the tarsal plate. The mesodermal tissue at the inferior border thickens and develops into fibrous tissue and extends to the inferior orbital margin as the septum orbitale. The medial and lateral collateral ligaments develop from the mesodermal tissue adjacent to the medial and lateral aspects of the tarsal plate $(80 \mathrm{~mm})$ and fuse with the adjacent periorbita of the medial and lateral tubercles respectively.

The anlage of the meibomian glands are not noted until $40 \mathrm{~mm}$. At this stage of development, epithelial buds from the lid margin develop in the flattened fibrous tissue of the tarsal plate. By a process of differential degeneration in the centre of this epithelial core, ducts develop from the lid margins and extend the length of the tarsal plate. By the $60 \mathrm{~mm}$ stage of development a central tube is present within the tarsal plate. By $100 \mathrm{~mm}$, the meibomian glands are lined by cuboidallike secreting epithelial.

The skin appendages develop from the twolayered epithelium lining the anterior surface of the lids. During the $35 \mathrm{~mm}$ stage of development, there are two layers of epithelium. The superficial layer is flattened and the deeper layer is cuboidal. During the $60 \mathrm{~mm}$ stage of development, three layers of cells develop from the basal cuboidal cells (the future stratum germinativum). Hair develops at this time. Keratin is present in the superficial layer of the epithelium $(150 \mathrm{~mm})$. The first crop of the lanugo hair covers the face and lids of the fetus $(160 \mathrm{~mm})$ (Fig. 8). This is shed just before and after birth. The lanugo hair in the region of the eyebrow, however, persists throughout life. Eyelashes are derived from the ingrowth of surface epithelium while the lids are fused ( $35 \mathrm{~mm}$ to $175 \mathrm{~mm}$ ). A solid core of epithelium grows into the anterior margin of the fused lid margins $(40 \mathrm{~mm})$ and later hair shafts are noted in the follicles $(90 \mathrm{~mm})$. The glands of Moll and Zeis also develop from epithelial ingrowths $(110 \mathrm{~mm})$.

The tarsal conjunctiva develops from a single layer of epithelium on the posterior surface of the lids and becomes mucous membrane $(50 \mathrm{~mm})$. Goblet cells develop in the conjunctiva after the lids have separated $(200 \mathrm{~mm})$. The accessory lacrimal glands of Krause are developed by epithelial ingrowths of the conjunctiva at about the 50 to $60 \mathrm{~mm}$ stage of development. These epithelial cores canalise by $110 \mathrm{~mm}$.

The puncta and canaliculi develop from a core of superficial epithelium that becomes $(50 \mathrm{~mm})$ buried below the surface of the upper

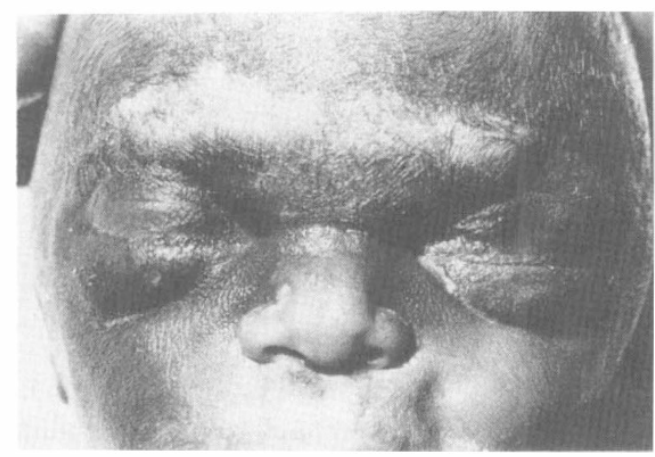

Fig. 8. Lanugo covering face, 5 months fetus. 
and the lower lids occupying the medial $1 / 6$ of these structures (Fig. 9). ${ }^{8}$ The trailing epithelial cells between the surface epithelium and the anlage of the future canaliculus atrophy. There is rapid growth of the maxilla compared to that of the frontal bone and the inferior canaliculus becomes stretched laterally. ${ }^{9}$ The inferior punctum therefore lies lateral to the superior punctum. Canalisation of the puncta and the canaliculi occurs between the 60 and $65 \mathrm{~mm}$ stage of development.

Prior to the lids forming, the optic vesicle and therefore the cornea are exposed to the amniotic fluid. In embryos ranging in length from $13.6 \mathrm{~mm}$ to $28.0 \mathrm{~mm}$, the corneal epithelium, which is 3 to 4 cells depth, is noted to be continuous with the surface ectoderm. Once the lids have fused $(35.0 \mathrm{~mm})$, the epithelial layer is reduced to a two layer structure. Only in the $210 \mathrm{~mm}$ fetus does the corneal epithelium become a 4 to 5 layered structure as is recognised in the term fetus.

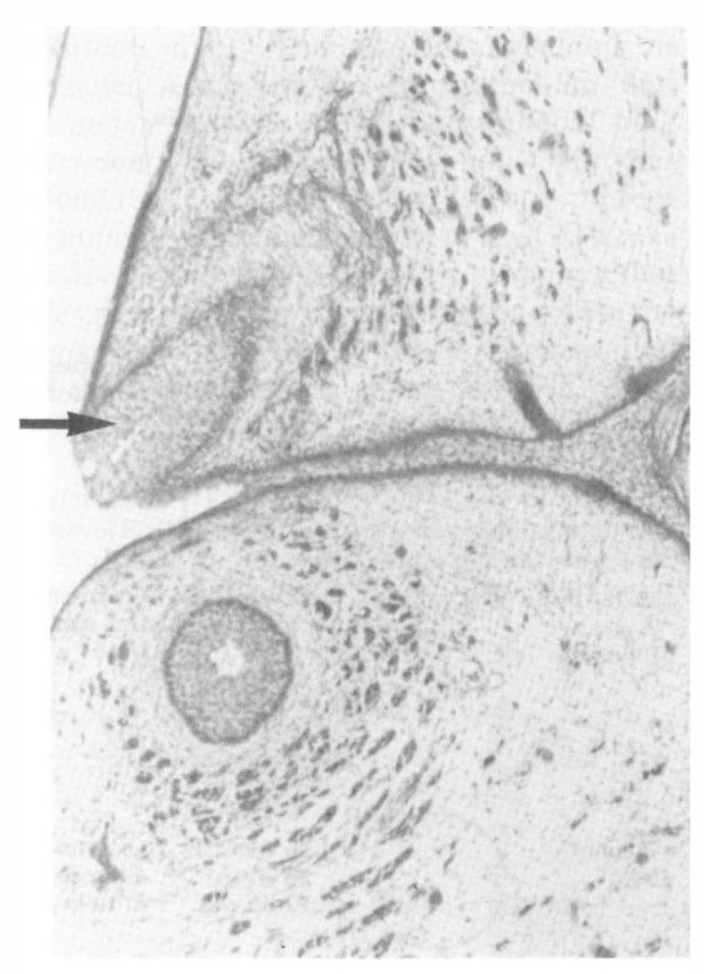

Fig. 9. Core of buried epithelium (arrow) forms from the superior and inferior canaliculi (open arrow) $(60 \mathrm{~mm}$, Heamatoxylin-eosin $\times 20)$.

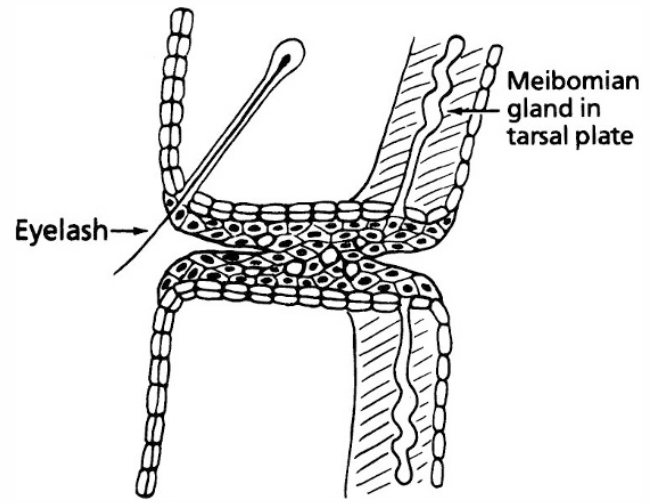

Fig. 10. Diagram showing separation of lids occurring on the anterior and posterior surfaces of the epithelial plug.

Stage of Eyelid Separation $(175 \mathrm{~mm}$ $250 \mathrm{~mm}$, 5 months to 7 months). (Fig. 10).

The lids start separating at about the $175 \mathrm{~mm}$ stage of development beginning nasally and extending temporally. The epithelial plug (formed by two epithelial layers of the upper lid and two layers of epithelium from the lower lid) commence to keratinise first on the epithelial surface and then on the conjunctival surface (Fig. 11). This causes erosion of the epithelial plug. In addition areas of degeneration occur in epithelial cells in the plug. This is evidenced by fragmentation of the nuclei eventually forming dust-like particles. The cytoplasm changes from an eosinophilic to a heamatoxylin stain and by $300 \mathrm{~mm}$ stage of development vesicles are noted in the epithelial plug. With this constant erosion, and cellular degeneration, separation of the lids

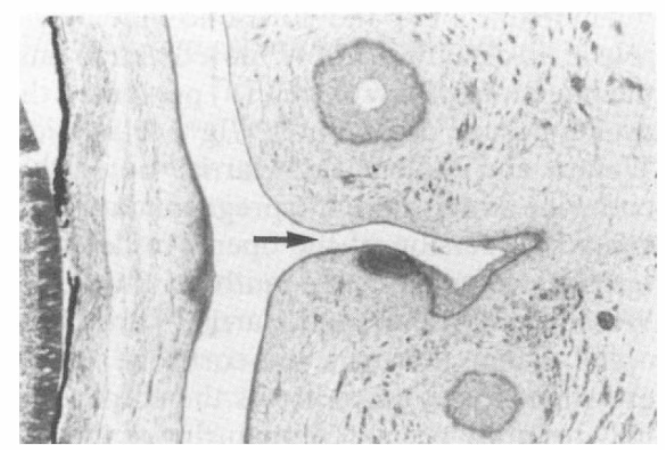

Fig. 11. Separation of lids (arrow) commences with keratinisation of epithelial surface $(200 \mathrm{~mm}$, Heamatoxylin-eosin $\times 3$ ). 
occurs. Fine elongated epithelial strands remain between the lids to term.

\section{Stage of Eyelid Maturation $(250 \mathrm{~mm}$ to term).}

Eyelid movement occurs about the $250 \mathrm{~mm}$ stage of development when nerve fibres in the orbicularis oculi and levator palpebrae superioris muscles are noted to be mature. The lanugo hair degenerates except for the region of the eyebrows. The lid margins, at the site of the remaining interpalpebral strands, become smooth. The epithelial plugs in the meibomian ducts disintegrate and meibomian secretions are noted just before term.

\section{Discussion}

Amniotic fluid may play a role in the development of the lids, although this has not been proved scientifically. Up to about the $35 \mathrm{~mm}$ stage of development the optic vesicle is exposed to the amniotic fluid in which there is a high concentration of sodium chloride. However, at about 12 weeks $\left(70 \mathrm{~mm},{ }^{10}\right.$ the kidney starts functioning and while the concentration of sodium chloride commences to decrease, the urea, uric acid and creatinine content of the amniotic fluid increases. ${ }^{11}$ The lids fuse $(35 \mathrm{~mm})$ prior to renal function commencing $(70 \mathrm{~mm})$. It is during the phase of lid fusion that the cornea matures developing five layers of epithelium, Bowman's membrane and Descemet's membrane so that by the time the lids separate the cornea is well protected from the renal excretory contents within the amniotic fluid. (Fig. 12).

Thyroxine (T4) and cortisone may play a role in lid closure. Juriloff ${ }^{12}$ noted that in rats, the injection off thyroxine (T4) prevented the eyelid closure defect in $\mathrm{Ig}^{\mathrm{m} 1} / \mathrm{Ig}^{\mathrm{m} 1}$ fetal mice. Watney and Miller ${ }^{13}$ and Harris ${ }^{14}$ noted that cortisone administered to pregnant dams prevented expression of the open eye defect in $\mathrm{Ig}^{\mathrm{m} 1} / \mathrm{Ig}^{\mathrm{m} 1}$ fetuses. These authors (Juriloff, ${ }^{12}$ Watney and Miller ${ }^{13}$ and Harris, $\left.{ }^{14}\right)$ are of the opinion that thyroxine or cortisone causes growth of the eye lids across the cornea and may result in 'premature maturing of the epidermis.' There is no clinical evidence in the human being to show that there is an increase of thyroxine or cortisone in amniotic fluid during the stages of the formation of the eye lid fold $(15 \mathrm{~mm}-35 \mathrm{~mm})$ or during the stage of eye lid fusion $(35 \mathrm{~mm}-175 \mathrm{~mm})$. During the phase of lid closure, the cornea matures in terms of developing five layers of epithelium a Bowman's membrane, thickened stroma, Descemet's membrane, and an endothelial layer.

Separation of lids occurs over a protracted period of time $[175 \mathrm{~mm}$ (5th month) to $250 \mathrm{~mm}$ (7th month)]. The mechanism of eye lid separation is not well understood. During this period of separation, keratinisation of the epithelium at the junction of the upper and lower lids commences. The process is noted initially at the medial canthal region. Triangular defects of keratinisation and degeneration occur at the fusional site both on the epithelial and on the conjunctival surfaces. As the process continues, the lid margins separate. It is difficult to assess the role of the levator palpebrae superioris muscle in the separation of

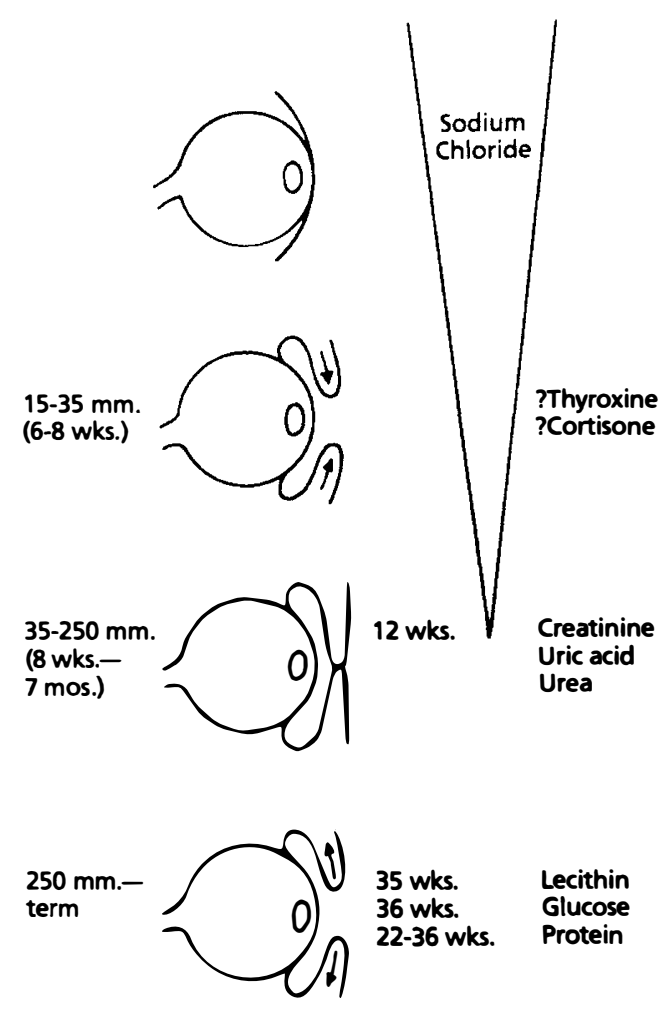

Fig. 12. A diagrammatic representation of lid development correlated with substances in the amniotic fluid. 
the lids. While the muscle is distinguishable in its development as early as $54 \mathrm{mms}$, cross striations are not noted until the $80 \mathrm{~mm}$ sized fetus. The inverted D-shaped tarsal plate becomes discernable about the same time. This may account for the levator palpebrae superioris muscle contracting, 'elongating' and therefore moulding the malleable tarsal plate to which it is adherant not only to the upper convex border but to its anterior surface as well. During the period of fusion of the lids, the meibomian glands develop and by the time the lids are separated $(250 \mathrm{~mm})$ acini are present in the glands. While no lytic substances have been demonstrated in meibomian secretions, which would aid lid separation, its oily consistency would certainly assist in maintaining the separation of the lids.

Cohen, ${ }^{15,16}$ having isolated a protein (epidermal growth factor) from the submaxillary gland of the rat, noted that it caused premature opening of the lids in the rat as early as seven days instead of the normal twelve to fourteen days. This protein appeared to enhance keratinisation of epithelium and caused a breakdown in the epithelial plug between the upper and lower lids. Orth ${ }^{17}$ has found that epidermal growth factor is present in human amniotic fluid from at least 20 weeks of development. It is tempting to suggest that the epidermal growth factor plays a role in the separation of the lids by causing keratnisation of the epithelial cells at the junction of the upper and lower lids.

\footnotetext{
References

1 Sevel D: The origins and insertion of the extraocular muscle. Development, Histological fatures, and
}

clinical significance. Tr Am Ophthalmol Soc 1986, 84: 488-526.

2 Sevel D: A reappraisal of the origin of human extraocular muscles. Ophihalmology 1981, 88: 1330-8.

${ }^{3}$ Arey LB: Developmental Anatomy. Philadelphia: WB Saunders 1942: 129-133.

${ }^{4}$ Tuchmann-duPlessis $H$, David G, Haegel P: Embryogenesis Illustrated Human Embryology. London: Chapman and Hall 1972: 88-91.

${ }^{5}$ Sevel D: Development of the nerves of the extraocular muscles. In Reinecke RD, ed. Strabismus. New York: Grune and Stratton 1984: 645-57.

6 Sevel D: Ptosis and underaction of the superior rectus muscle. Ophthalmology 1983, 91: 1080-5.

${ }^{7}$ Sevel D: Brown's Syndrome. A possible etiology explained embryologically. Ophthalmology 1981, 18: 26-31.

${ }^{8}$ Sevel D: Development and congenital abnormalities of the nasolacrimal apparatus. J Ped Ophthalmol and Strab 1981, 18: 13-9.

${ }^{9}$ Sevel D: Insufflation treatment of occluded nasolacrimal apparatus. J Ped Ophthalmol and Strab 1982, 89: 329-34.

${ }^{10}$ Hewer EE: Secretion by the human foetal kidney. Qart J Exp Pysiol 1924, 14: 1924.

11 Seeds AE: Water metabolism of the foetus. Am J Obstet Gynecol 1965, 92: 727.

12 Juriloff DM: Prevention of the eye closure defect in Igm1/Igm1 J Teratol 1985, 32: 3-86.

${ }^{13}$ Watney MJ and Miller JR: Prevention of a genetically determined congenital eye anomaly in the mouse by the administration of cortisone during pregnancy. Nature 1964, 202: 1029-31.

${ }^{14}$ Harris MJ, Juriloff DM, Biddle B: Cortisone cure of the lidgap defect in fetal mice. A dose-response and time-response study. Teratology 1984, 29: 287-95.

${ }^{15}$ Cohen S: Isolation of a mouse submaxillary gland, protein accelerating incisor eruption and eyelid opening in the newborn animal. J Biol Chem 1962, 237: 1555-62.

${ }^{16}$ Cohen S and Elliott GA: The stimulation of epidermal keratinisation by a protein isolated from the submaxillary gland of the mouse. J Invest Derm 1963, 40: $1-5$.

${ }^{17}$ Orth D: Personal communication 1987. 